\title{
FAST EVALUATION OF MODULAR FUNCTIONS USING NEWTON ITERATIONS AND THE AGM
}

\author{
RÉGIS DUPONT
}

\begin{abstract}
We present an asymptotically fast algorithm for the numerical evaluation of modular functions such as the elliptic modular function $j$. Our algorithm makes use of the natural connection between the arithmetic-geometric mean (AGM) of complex numbers and modular functions. Through a detailed complexity analysis, we prove that for a given $\tau$, evaluating $N$ significative bits of $j(\tau)$ can be done in time $O(\mathcal{M}(N) \log N)$, where $\mathcal{M}(N)$ is the time complexity for the multiplication of two $N$-bit integers. However, this is only true for a fixed $\tau$ and the time complexity of this first algorithm greatly increases as $\operatorname{Im}(\tau)$ does. We then describe a second algorithm that achieves the same time complexity independently of the value of $\tau$ in the classical fundamental domain $\mathcal{F}$. We also show how our method can be used to evaluate other modular forms, such as the Dedekind $\eta$ function, with the same time complexity.
\end{abstract}

\section{INTRODUCTION}

In this article, we study the complexity of the numerical evaluation of modular functions at complex values: given an element $\tau$ lying in $\mathcal{F}=\{z \in \mathbb{C}: \operatorname{Im}(z)>0$, $|z| \geq 1,|\operatorname{Re}(z)| \leq 1 / 2\}$ and an integer $N \geq 0$, we investigate the time complexity for the evaluation of $N$ significative bits of modular functions such as $j$ at $\tau$. Our main result is a constructive proof of the following theorem.

Theorem. Given an integer $N \geq 0$ and an element $\tau \in \mathcal{F}$, one can compute $J \in \mathbb{C}$ such that

$$
\left|\frac{j(\tau)-J}{j(\tau)}\right| \leq \frac{1}{2^{N}}
$$

in time $O(\mathcal{M}(N) \log N)$, where $\mathcal{M}(N)$ is the time complexity for the multiplication of two $N$-bit integers 1

This work was motivated in part by applications such as the computation of Hilbert class polynomials [7] and the computation of modular polynomials [8], where it can be necessary to evaluate modular functions to precision in the tens of thousands of bits.

We begin by recalling a few facts about modular functions and theta constants before giving a first (naïve) algorithm for the evaluation of a class of modular functions with a running time in $O(\mathcal{M}(N) \sqrt{N})$, where $N$ is the required precision. Then

Received by the editor May 27, 2005.

2000 Mathematics Subject Classification. Primary 65D20; Secondary 33E05, 11 Y16.

Key words and phrases. Modular functions, numerical evaluation, AGM, Newton iterations.

${ }^{1}$ Using FFT-based multiplication, we typically have $\mathcal{M}(N)=O(N \log N \log \log N)=O\left(N^{1+\epsilon}\right)$.

(C)2011 Régis Dupont 
we study some properties of the arithmetic-geometric mean (AGM), show how it can be used to evaluate a particular modular function using Newton iterations and give a first algorithm in which time complexity is thoroughly analyzed. This first algorithm achieves the announced complexity in $O(\mathcal{M}(N) \log N)$ but only in the case where $\tau$ is fixed. We then propose a second algorithm having the same running time, independently of the value of $\tau \in \mathcal{F}$. Finally, we show how this method can be used to evaluate other modular functions or forms, such as the Dedekind $\eta$ function, and we give some experimental results.

An important feature of our algorithm is that it can be generalized to genus 2 (see the forthcoming [6]).

\section{Modular FunCtions AND theta CONSTANTS}

2.1. Modular functions. In this section, we briefly recall a few facts about modular functions and fix some notation. We refer the reader to [15] for a complete introduction to the theory of modular functions.

Throughout this paper, we let $\mathcal{H}$ denote the Poincaré half-plane $\mathcal{H}=\{z \in \mathbb{C}$ : $\operatorname{Im}(z)>0\}$. For $\tau \in \mathcal{H}$, we let $q=q(\tau)=\exp (i \pi \tau)$ (note that this differs from modern conventions, where an extra factor of 2 is added). We denote by $\Gamma$ the full elliptic modular group, that is the quotient of $\mathrm{SL}_{2}(\mathbb{Z})$ by $\langle-I\rangle$, and from now on we identify a matrix in $\mathrm{SL}_{2}(\mathbb{Z})$ with its class in $\Gamma$. The action of $\Gamma$ on $\mathcal{H}$ is given by

$$
\left(\begin{array}{ll}
a & b \\
c & d
\end{array}\right) \tau=\frac{a \tau+b}{c \tau+d},
$$

for every $\left(\begin{array}{ll}a & b \\ c & d\end{array}\right) \in \Gamma$ and $\tau \in \mathcal{H}$.

If we introduce the region $\mathcal{F} \subset \mathcal{H}$,

$$
\mathcal{F}=\{\tau \in \mathcal{H}:|\operatorname{Re}(\tau)| \leq 1 / 2,|\tau| \geq 1\},
$$

then $\mathcal{F} \backslash(\partial \mathcal{F} \cap\{\tau \in \mathcal{H}: \operatorname{Re}(\tau)>0\})$ is a fundamental domain for the action of $\Gamma$ on $\mathcal{H}$.

We also introduce the generators $S$ and $T$ of $\Gamma$, defined by

$$
S=\left(\begin{array}{cc}
0 & -1 \\
1 & 0
\end{array}\right)
$$

and

$$
T=\left(\begin{array}{ll}
1 & 1 \\
0 & 1
\end{array}\right),
$$

so that for every $\tau \in \mathcal{H}, T \tau=\tau+1$ and $S \tau=-1 / \tau$.

If $\Gamma^{\prime}$ is a subgroup of finite index of $\Gamma$, then a function $f: \mathcal{H} \rightarrow \mathbb{C}$ is said to be modular for $\Gamma^{\prime}$ if it is meromorphic on $\mathcal{H}$, invariant under the action induced by $\Gamma^{\prime}$ (i.e., for all $\gamma \in \Gamma^{\prime}$ and $\tau \in \mathcal{H}, f(\gamma \tau)=f(\tau)$ ), and meromorphic at the cusps.

2.2. Theta constants with characteristic 2 . The so-called theta constants with characteristic 2 are the three functions defined, for $\tau \in \mathcal{H}$ and $q=\exp (i \pi \tau)$, by

$$
\begin{gathered}
\theta_{00}(\tau)=\sum_{n \in \mathbb{Z}} q^{n^{2}}, \\
\theta_{01}(\tau)=\sum_{n \in \mathbb{Z}}(-1)^{n} q^{n^{2}},
\end{gathered}
$$


and

$$
\theta_{10}(\tau)=\sum_{n \in \mathbb{Z}} q^{\left(n+\frac{1}{2}\right)^{2}}
$$

For an extensive treatment of these functions, we refer the reader to [14] or [16], which contain the proofs of all the few properties we now state (we only give proofs when elementary ones exists, that we are aware of).

Proposition 1. The functions $\theta_{00}, \theta_{01}$, and $\theta_{10}$ are analytic on $\mathcal{H}$ and do not vanish.

Proof. That these functions are analytic on $\mathcal{H}$ follows from their definition as $q$ series. That they do not vanish on $\mathcal{H}$ follows from [14, Lemma 4.1].

Proposition 2. For all $\tau \in \mathcal{H}$,

$$
\begin{aligned}
& \theta_{00}^{2}(S \tau)=-i \tau \theta_{00}^{2}(\tau) \\
& \theta_{01}^{2}(S \tau)=-i \tau \theta_{10}^{2}(\tau) \\
& \theta_{10}^{2}(S \tau)=-i \tau \theta_{01}^{2}(\tau)
\end{aligned}
$$

Proof. See [16, pp. 103-105].

Proposition 3. For all $\tau \in \mathcal{H}$,

$$
\theta_{00}^{4}(\tau)=\theta_{01}^{4}(\tau)+\theta_{10}^{4}(\tau)
$$

Proof. See [16, pp. 71-76].

\section{Proposition 4.}

$$
\begin{array}{r}
\lim _{\operatorname{Im}(\tau) \rightarrow+\infty} \theta_{00}(\tau)=1, \\
\lim _{\operatorname{Im}(\tau) \rightarrow+\infty} \theta_{01}(\tau)=1,
\end{array}
$$

and

$$
\lim _{\operatorname{Im}(\tau) \rightarrow+\infty} \theta_{10}(\tau)=0 .
$$

Proof. This is easily proven from the definitions of the theta functions as $q$-series.

Proposition 5. For all $\tau \in \mathcal{H}$,

$$
\theta_{00}^{2}(2 \tau)=\frac{\theta_{00}^{2}(\tau)+\theta_{01}^{2}(\tau)}{2}
$$

and

$$
\theta_{01}^{2}(2 \tau)=\theta_{00}(\tau) \theta_{01}(\tau)
$$

Proof. This is easily derived from the definitions of $\theta_{00}$ and $\theta_{01}$, using the identity $2\left(n^{2}+m^{2}\right)=(n+m)^{2}+(n-m)^{2}$.

Proposition 6. For all $\tau \in \mathcal{H}$,

$$
\begin{aligned}
& 4 \frac{d}{d \tau} \log \frac{\theta_{10}}{\theta_{01}}(\tau)=i \pi \theta_{00}^{4}(\tau), \\
& 4 \frac{d}{d \tau} \log \frac{\theta_{00}}{\theta_{01}}(\tau)=i \pi \theta_{10}^{4}(\tau), \\
& 4 \frac{d}{d \tau} \log \frac{\theta_{10}}{\theta_{00}}(\tau)=i \pi \theta_{01}^{4}(\tau) .
\end{aligned}
$$


Proof. See [16, p. 82].

2.3. The functions $k$ and $k^{\prime}$. Jacobi's functions $k$ and $k^{\prime}$ are defined, for $\tau \in \mathcal{H}$, by

$$
k(\tau)=\left(\frac{\theta_{10}(\tau)}{\theta_{00}(\tau)}\right)^{2}
$$

and

$$
k^{\prime}(\tau)=\left(\frac{\theta_{01}(\tau)}{\theta_{00}(\tau)}\right)^{2} .
$$

From Propositions 2 and 3 , it follows directly that, for all $\tau \in \mathcal{H}$,

- $k^{2}(\tau)+k^{2}(\tau)=1$,

- $k(S \tau)=k^{\prime}(\tau)$ and $k^{\prime}(S \tau)=k(\tau)$.

Moreover, $k^{\prime}$ and $k$ are modular functions:

Proposition 7. The functions $k^{\prime}$ and $k$ are modular for the groups $\Gamma_{k^{\prime}}$ and $\Gamma_{k}$, where

$$
\Gamma_{k^{\prime}}=\left\{\left(\begin{array}{cc}
a & b \\
c & d
\end{array}\right) \in \Gamma: a \equiv d \equiv 1 \bmod 4, b \equiv 0 \bmod 2, c \equiv 0 \bmod 4\right\}
$$

and

$$
\Gamma_{k}=\left\{\left(\begin{array}{cc}
a & b \\
c & d
\end{array}\right) \in \Gamma: a \equiv d \equiv 1 \bmod 4, b \equiv 0 \bmod 4, c \equiv 0 \bmod 2\right\} .
$$

Moreover, if

$$
\mathcal{F}^{\prime}=\{\tau \in \mathcal{H}:|\operatorname{Re}(z)|<1,|2 \tau+1|>1,|2 \tau-1|>1\},
$$

then

$$
k^{\prime}\left(\mathcal{F}^{\prime}\right)=k\left(\mathcal{F}^{\prime}\right)=\{z \in \mathbb{C} \backslash\{0,1\}: \operatorname{Re}(z)>0\},
$$

and the real part of $k(\tau)$ (resp. of $k^{\prime}(\tau)$ ) vanishes on the boundary of $\mathcal{F}^{\prime}$.

Proof. The results for $k^{\prime}$ are proved in [4, Lemmata 2.7 and 2.8]. The results for $k$ follow easily, using $k(\tau)=k^{\prime}(S \tau)$ and the identities $\Gamma_{k}=S \Gamma_{k^{\prime}} S$ and $S \mathcal{F}^{\prime}=\mathcal{F}^{\prime}$.

Finally, the well-known elliptic modular function $j$ (modular for the full group Г) can be defined by

$$
j(\tau)=256 \frac{\left(1-k^{\prime 2}(\tau)+k^{\prime 4}(\tau)\right)^{3}}{k^{\prime 4}(\tau)\left(1-k^{\prime 2}(\tau)\right)^{2}} .
$$

(see [2, pages 112-116] for a proof of the fact that $j$ is indeed modular for $\Gamma$ ).

\section{Basic evaluation of $j$ Using the Dedekind $\eta$-Function}

3.1. Notions of precision. Our goal in this paper is to evaluate some functions with a given precision. We now define precisely what we mean by precision:

- We will say that $\alpha \in \mathbb{C}$ is an approximation of $a \in \mathbb{C}$ with absolute precision $N$ bits if

$$
|a-\alpha| \leq \frac{1}{2^{N}} .
$$


- We will say that $\alpha \in \mathbb{C}$ is an approximation of $a \in \mathbb{C}$ with relative precision $N$ bits if

$$
\left|\frac{a-\alpha}{a}\right| \leq \frac{1}{2^{N}}
$$

We will make use of the following facts, whose proofs are straightforward:

- If $\alpha$ and $\beta$ are approximations of $a$ and $b \in \mathbb{C}$ with relative precision $N$ bits $(N \geq 2)$, then $\alpha \beta$ is an approximation of $a b$ with relative precision $N-2$ bits.

- If $n \geq 1$ and $\alpha$ is an approximation of $a \in \mathbb{C}$ with relative precision $N$ bits $(N \geq n)$, then $\alpha^{n}$ is an approximation of $a^{n}$ with relative precision $N-$ $n$ bits.

- If $\alpha \neq 0$ is an approximation of $a \in \mathbb{C} \backslash\{0\}$ with relative precision $N$ bits $(N \geq 1)$, then $\alpha^{-1}$ is an approximation of $a^{-1}$ with relative precision $N-1$ bits.

- If $\alpha$ is an approximation of $a \in \mathbb{C}$ with relative precision $N$ bits, and if $\sqrt{a}$ is any of the two square roots of $a$, then the square root of $\alpha$ lying in the same quarter-plane as $\sqrt{a}$ is an approximation of $\sqrt{a}$ with relative precision $N$ bits.

- If $\alpha$ and $\beta$ are approximations of $a$ and $b \in \mathbb{C}$ with absolute precision $N$ bits $(N \geq 1)$, then $\alpha+\beta$ is an approximation of $a+b$ with absolute precision $N-1$ bits.

One of the most delicate issues when studying algorithms to numerically evaluate functions is to know to what precision each computation has to be carried out. Due to what precedes, we know that precision losses will occur, hence the working precision will usually have to be higher than the precision required for the result. However, since the time cost of arithmetic operations increases with the working precision, one should always work at the minimal possible precision ensuring that the final result will be correct to the required precision.

In what follows, the precision at which arithmetic operations have to be carried out is not specified in the algorithms we give, but is discussed after the description of these algoritms.

3.2. A naïve algorithm. It is well known that the function $j$ has a $q$-series expansion with integral coefficients:

$$
j(\tau)=\frac{1}{q^{2}}+744+169884 q^{2}+\cdots,
$$

the coefficients of this series being effectively computable (see for example [1]).

However, this leads to an algorithm that is quite inefficient, since the series we consider is dense and the height of its coefficients grows quite fast: the time complexity would be in $O(\mathcal{M}(N) N)=O\left(N^{2+\varepsilon}\right)$, where $N$ is the required precision in bits.

A better approach is to use the functions $\theta_{00}$ and $\theta_{01}$ : their $q$-series can indeed be evaluated much faster than the one for $j$, since they are sparse with non-nil coefficients \pm 1 . The function $k^{\prime}$ can be computed as the square of their quotient, and $j$ can then be evaluated using equation (3).

For $\varepsilon \in\{0,1\}$ and $B \geq 1$, we define the partial sums

$$
S_{\varepsilon, B}(\tau)=\left\{\begin{array}{lll}
1, & \text { if } & B=1, \\
1+2 \sum_{n=1}^{B-1}(-1)^{\varepsilon n} q^{n^{2}}, & \text { if } & B \geq 2,
\end{array}\right.
$$

for $\tau \in \mathcal{F}$. 
Notice that if $\tau \in \mathcal{F}$, then $\operatorname{Im}(\tau) \geq \sqrt{3} / 2$, hence $|q| \leq Q=\exp (-\pi \sqrt{3} / 2)$. Thus, using the inequality

we obtain

$$
\left|\sum_{n \geq B} q^{n^{2}}\right| \leq \frac{|q|^{B^{2}}}{1-|q|} \leq 2|q|^{B^{2}}
$$

$$
\left|\theta_{0 \varepsilon}(\tau)-S_{\varepsilon, B}(\tau)\right| \leq 4|q|^{B^{2}} .
$$

Notice that in the case $B=1$, this implies

$$
\left|\theta_{0 \varepsilon}(\tau)-1\right| \leq 4|q| \leq 4 Q \leq 0.27,
$$

hence $\theta_{0 \varepsilon}(\tau)$ is "close" to 1 : for all $\tau \in \mathcal{F}$, any approximation of $\theta_{0 \varepsilon}$ with absolute precision $N \geq 1$ bits is also an approximation with relative precision $N-1$ bits.

Now a straightforward computation shows that for all $\tau \in \mathcal{F}, N \in \mathbb{N}$ and $\varepsilon \in$ $\{0,1\}$, if

then

$$
B \geq \sqrt{\frac{N+3}{\pi \operatorname{Im}(\tau) \log _{2} e}},
$$

for $\varepsilon \in\{0,1\}$.

$$
\left|\frac{\theta_{0 \varepsilon}(\tau)-S_{\varepsilon, B}(\tau)}{\theta_{0 \varepsilon}(\tau)}\right| \leq \frac{1}{2^{N}}
$$

This shows that Algorithm 1 can be used to evaluate $\theta_{00}$ and $\theta_{01}$.

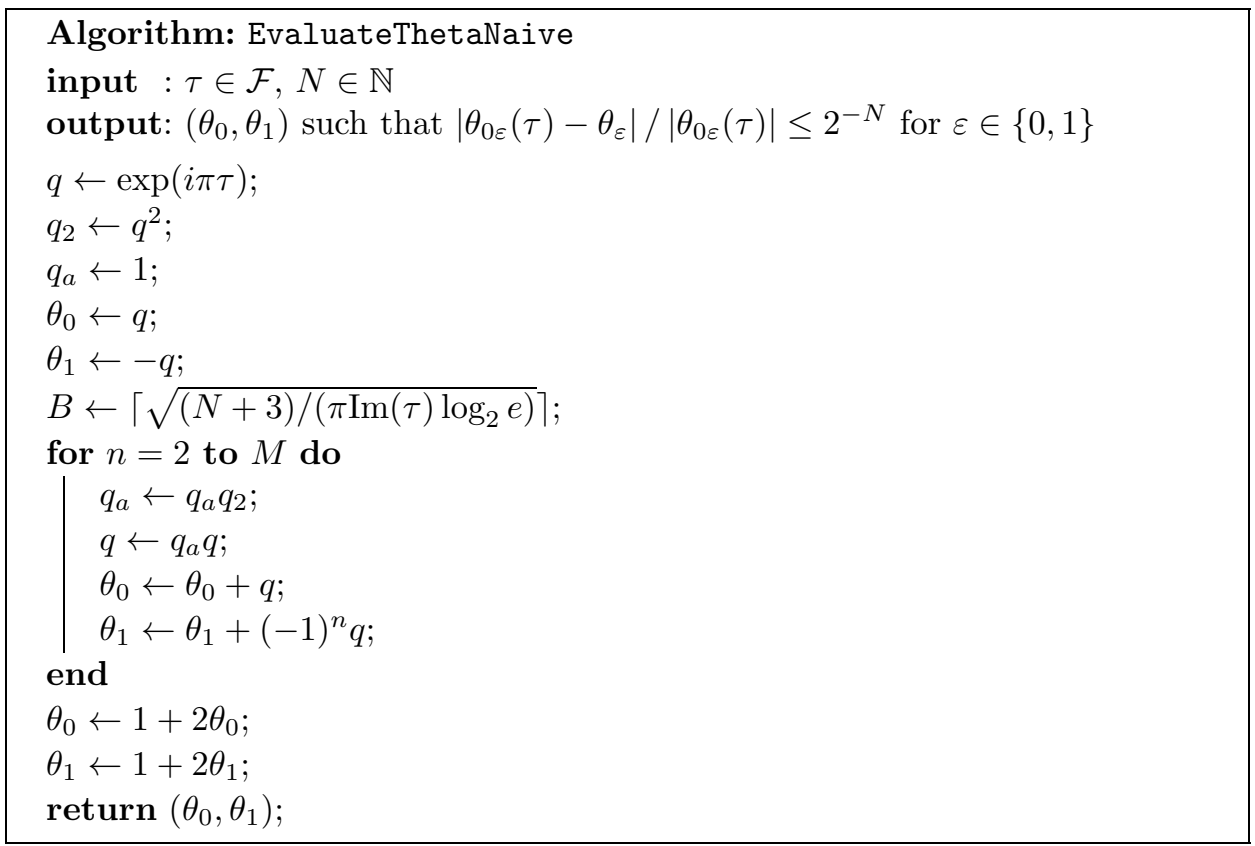

Algorithm 1: Naïve evaluation of $\theta_{00}$ and $\theta_{01}$

We now discuss the precision at which the computations have to be carried out. From what we have seen before, the final result should be correct with absolute precision at least $N+1$ bits. Clearly, this will be achieved if all computations are carried out at fixed precision $N+5 \log _{2} M=O(N)$ bits. Since the constant $\pi$ as 
well as the exponential function can be evaluated with relative precision $N$ bits in time $O(\mathcal{M}(N) \log N)$, as explained in [3] for example, the time complexity of algorithm EvaluateThetaNaive (Algorithm 1) is in $O(\mathcal{M}(N) \sqrt{N})$.

We now turn to the evaluation of $k^{\prime}$ : since $k^{\prime}(\tau)=\left(\frac{\theta_{01}(\tau)}{\theta_{00}(\tau)}\right)^{2}$, and considering the precision losses that can occur, Algorithm 2 can be used to evaluate $k^{\prime}$.

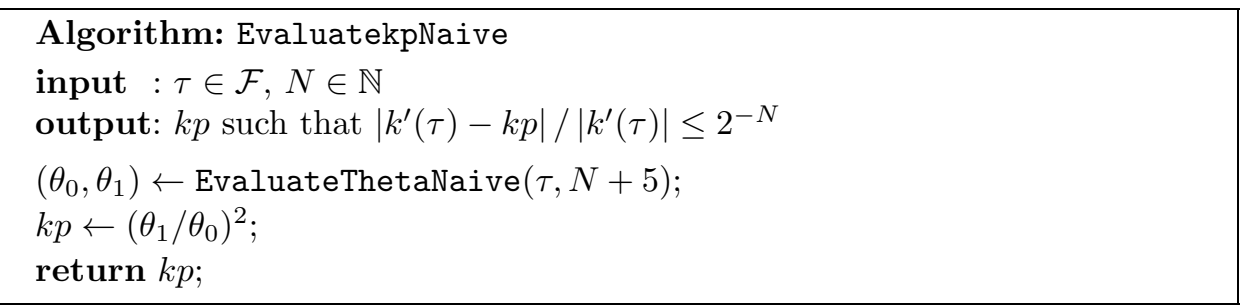

Algorithm 2: Naïve evaluation of $k^{\prime}$

Clearly, algorithm EvaluatekpNaive (Algorithm 2) has time complexity in $O(\mathcal{M}(N) \sqrt{N})$.

Now $j$ can be evaluated using equation (3). However, one must be careful about the precision loss that can occur: as $\operatorname{Im}(\tau)$ increases, $\theta_{00}(\tau)$ and $\theta_{01}(\tau)$ will tend to 1 , hence $1-k^{\prime}(\tau)^{2}$ will tend to zero. More precisely, we have $1-k^{\prime 2}(\tau) \sim 16 q$ as $\operatorname{Im}(\tau) \rightarrow+\infty$, hence this operation can entail a big precision loss. In fact, in order to evaluate $j(\tau)$ with relative precision $N$ bits, it is sufficient to have an evaluation of $k^{\prime}(\tau)$ with relative precision $N+5 \operatorname{Im}(\tau)$ bits, and the algorithm is of course straightforward.

Remark 1. The $j$ function can also be defined by means of the Dedekind $\eta$ function, which shares a few properties with theta constants (sparse $q$ series development,...). It then follows that using the $\eta$ function $q$-series instead of those of the theta constants yields another algorithm for the evaluation of $j$ and related functions. This algorithm is described in [7] and has the same asymptotic complexity (up to a constant) as EvaluatekpNaive.

Remark 2. At first sight, the complexity of this method for the evaluation of $j$ depends on the value of $\operatorname{Im}(\tau)$. Note, however, that the number $B$ of iterations in Algorithm 1 is in $\sqrt{N / \operatorname{Im}(\tau)}$. In particular, if we fix some constant $K>0$ and we want to evaluate the theta constants at $\tau \in \mathcal{F}$ with relative precision $N \leq K \operatorname{Im}(\tau)$ bits, then the number of iterations will be bounded by a constant. Using this remark, it is easy to see that the complexity of the evaluation of $j$ is in $O(\mathrm{M}(N) \sqrt{N})$.

\section{THE ARITHMETIC-GEOMETRIC MEAN}

4.1. Definition. Let $a$ and $b$ be two positive real numbers. The arithmetic-geometric mean (AGM) of $a$ and $b$, uncovered by Lagrange and then rediscovered and much studied by Gauss, is the common limit of the two sequences $\left(a_{n}\right)$ and $\left(b_{n}\right)$ defined by $a_{0}=a, b_{0}=b$,

and

$$
a_{n+1}=\frac{a_{n}+b_{n}}{2}
$$

$$
b_{n+1}=\sqrt{a_{n} b_{n}} .
$$


A proof of the fact that these sequences indeed converge to a common limit can be found in [11, III, pp. 361-363] or in [2, 4].

The AGM can be extended to the case where $a$ and $b$ are complex numbers, although such a generalization is not straightforward (this is mainly due to the fact that, for every $n \in \mathbb{N}, b_{n+1}$ is defined as being one of the two possible square roots of $a_{n} b_{n}$, hence the sequences $\left(a_{n}\right)$ and $\left(b_{n}\right)$ are not uniquely determined by $a$ and $b$ ). A study of the AGM for complex numbers can again be found in [11, III, pp. 361-480] or in [4.

In order to simplify things somewhat, we only consider the case where $a$ and $b$ lie in $\mathcal{R}=\{z \in \mathbb{C}: \operatorname{Re}(z)>0\}$. For $z \in \mathbb{C}$, we denote by $\sqrt{z}$ the unique square root of $z$ lying in $\mathcal{R} \cup \mathbb{R}^{\geq 0}$.

We then define the sequences $\left(a_{n}\right)$ and $\left(b_{n}\right)$ as above. We have the following result.

Theorem 1. Let $a, b \in \mathcal{R}$, and define the sequences $\left(a_{n}\right)$ and $\left(b_{n}\right)$ as above. Then they converge to a common limit $\operatorname{AGM}(a, b) \in \mathcal{R}$, and this convergence is quadratic: for all $n \in \mathbb{N}$,

$$
\left|a_{n+1}-b_{n+1}\right| \leq \frac{\pi\left|a_{n}-b_{n}\right|^{2}}{8 \operatorname{Min}(|a|,|b|)} .
$$

Proof. For every $n \in \mathbb{N}$, let $\theta_{n} \in\left[0, \pi\left[\right.\right.$ denote the unoriented angle between $a_{n}$ and $b_{n}$. Proposition 2.1 of [4] proves that $\left(a_{n}\right)$ and $\left(b_{n}\right)$ converge to a common limit $\operatorname{AGM}(a, b) \in \mathcal{R}$, and the proof also shows that

$$
\left|a_{n+1}-b_{n+1}\right| \leq \frac{\left|a_{n}-b_{n}\right|}{2}
$$

and

$$
\theta_{n+1} \leq \frac{\theta_{n}}{2}
$$

For $n \in \mathbb{N}$, we have

$$
\begin{aligned}
\left|a_{n+1}-b_{n+1}\right| & =\frac{\left|a_{n}+b_{n}-2 \sqrt{a_{n} b_{n}}\right|}{2} \\
& =\frac{\left|\sqrt{a_{n}}-\sqrt{b_{n}}\right|^{2}}{2} \\
& =\frac{\left|a_{n}-b_{n}\right|^{2}}{2\left|\sqrt{a_{n}}+\sqrt{b_{n}}\right|^{2}} .
\end{aligned}
$$

Now, the unoriented angle between $\sqrt{a_{n}}$ and $\sqrt{b_{n}}$ is equal to $\theta_{n} / 2<\pi / 2$, so that

$$
\begin{aligned}
\left|\sqrt{a_{n}}+\sqrt{b_{n}}\right|^{2} & =\left|\sqrt{a_{n}}\right|^{2}+\left|\sqrt{b_{n}}\right|^{2}+2\left|\sqrt{a_{n} b_{n}}\right| \cos \left(\theta_{n} / 2\right) \\
& \geq\left|a_{n}\right|+\left|b_{n}\right| \\
& \geq 2 \operatorname{Min}\left(\left|a_{n}\right|,\left|b_{n}\right|\right) .
\end{aligned}
$$

From the proof of [4, Proposition 2.1], we also have that

$$
\operatorname{Min}\left(\left|a_{n}\right|,\left|b_{n}\right|\right) \geq \frac{\sin \theta_{0}}{\theta_{0}} \operatorname{Min}(|a|,|b|) \geq \frac{2}{\pi} \operatorname{Min}(|a|,|b|),
$$


whence

and finally

$$
\left|\sqrt{a_{n}}+\sqrt{b_{n}}\right|^{2} \geq \frac{4}{\pi} \operatorname{Min}(|a|,|b|)
$$

$$
\left|a_{n+1}-b_{n+1}\right| \leq \frac{\pi\left|a_{n}-b_{n}\right|^{2}}{8 \operatorname{Min}(|a|,|b|)} .
$$

The limit $\operatorname{AGM}(a, b)$ for $a$ and $b \in \mathcal{R}$, is again called the arithmetic-geometric mean of $a$ and $b$.

4.2. Relationship with theta constants and other properties. In order to simplify notations, we let $M: \mathcal{R} \rightarrow \mathcal{R}$ denote the (univariate) AGM 2 defined by $M(z)=\operatorname{AGM}(1, z)$.

The key point in the study of the AGM, relating it to modular functions and modular forms, is the following result.

Theorem 2. For $\tau \in \mathcal{F}^{\prime}$, we have

$$
M\left(k^{\prime}(\tau)\right)=\frac{1}{\theta_{00}^{2}(\tau)} .
$$

Proof. Let $\tau \in \mathcal{H}$, and define the sequences $\left(a_{n}\right)$ and $\left(b_{n}\right)$ by

$$
a_{n}=\frac{\theta_{00}^{2}\left(2^{n} \tau\right)}{\theta_{00}^{2}(\tau)}
$$

and

$$
b_{n}=\frac{\theta_{01}^{2}\left(2^{n} \tau\right)}{\theta_{00}^{2}(\tau)}
$$

Proposition 4 shows that

$$
\lim _{n \rightarrow \infty} \theta_{00}\left(2^{n} \tau\right)=\lim _{n \rightarrow \infty} \theta_{01}\left(2^{n} \tau\right)=1,
$$

whence

$$
\lim _{n \rightarrow \infty} a_{n}=\lim _{n \rightarrow \infty} b_{n}=\frac{1}{\theta_{00}^{2}(\tau)}
$$

Since $k^{\prime}(\tau)=\frac{\theta_{01}^{2}(\tau)}{\theta_{00}^{2}(\tau)}$, it is clear that $a_{0}=1$ and $b_{0}=k^{\prime}(\tau)$. Moreover, Proposition 5 proves that, for any $n \in \mathbb{N}$, we have

$$
a_{n+1}=\frac{a_{n}+b_{n}}{2}
$$

and

$$
b_{n+1}^{2}=a_{n} b_{n} .
$$

Hence the key point in obtaining the announced result is proving that, if $\tau \in \mathcal{F}^{\prime}$, then for any $n \in \mathbb{N}, a_{n}$ and $b_{n}$ lie in $\mathcal{R}$. This is contained in the proof of Theorem 2.2 of [4] and more precisely in the proof of Lemma 2.9, which also makes use of Lemmata 2.3 to 2.8 .

Proposition 8. The function $M$ is analytic on $\mathcal{R} \backslash\{1\}$.

\footnotetext{
${ }^{2}$ Considering that a univariate AGM makes sense, since the bivariate AGM is homogenous, for all $a, b \in \mathbb{C}, \operatorname{AGM}(a, b)=a \operatorname{AGM}(1, b / a)$.
} 
Proof. We first prove that the Inverse Function Theorem can be applied to the function $k^{\prime}$ at any point in $\mathcal{F}^{\prime}$. This is due to the fact that $k^{\prime}$ is analytic on $\mathcal{F}^{\prime}$, and that, for all $\tau \in \mathcal{F}^{\prime}$,

$$
\frac{d k^{\prime}}{d \tau}(\tau)=\frac{-i \pi \theta_{01}^{2}(\tau) \theta_{10}^{4}(\tau)}{2 \theta_{00}^{2}(\tau)} \neq 0
$$

(this expression of the derivative of $k^{\prime}$ is obtained using Proposition 6).

Now the function $1 / \theta_{00}^{2}$ is also analytic on $\mathcal{F}^{\prime}$, and for all $\tau \in \mathcal{F}^{\prime}$, we have

$$
M\left(k^{\prime}(\tau)\right)=\frac{1}{\theta_{00}^{2}(\tau)},
$$

hence the proposition follows from the fact that $k^{\prime}$ is surjective from $\mathcal{F}^{\prime}$ to $\mathcal{R} \backslash\{1\}$.

Proposition 9. For $\tau \in \mathcal{F}^{\prime}$, we have

$$
\frac{d M}{d z}\left(k^{\prime}(\tau)\right)=\frac{4 \theta_{00}^{\prime}(\tau)}{i \pi \theta_{00}(\tau) \theta_{01}^{2}(\tau) \theta_{10}^{4}(\tau)},
$$

where $\theta_{00}^{\prime}(\tau)=\frac{d \theta_{00}}{d \tau}(\tau)$.

Proof. This follows directly from the differentiation of the equality

$$
M\left(k^{\prime}(\tau)\right)=\frac{1}{\theta_{00}^{2}(\tau)},
$$

using the definition of the function $k^{\prime}$ and Proposition 6 .

\section{Evaluating $k^{\prime}$ using Newton iterations and the AGM}

In this section, we restrict our work to the case $\tau \in \mathcal{F}$. Using the same arguments as in Section 3, it is easy to see that for all $\tau \in \mathcal{F}$,

$$
\left|k^{\prime}(\tau)-1\right| \leq 0.9
$$

hence any approximation of $k^{\prime}(\tau)$ with absolute precision $N+4$ bits is also an approximation with relative precision $N$ bits.

5.1. A function vanishing at $k^{\prime}(\tau)$. Let $\tau \in \mathcal{F}$, since $\mathcal{F} \subset \mathcal{F}^{\prime}$ and $S \mathcal{F} \subset \mathcal{F}^{\prime}$, we have $k^{\prime}(\tau) \in \mathcal{R}$ and $k^{\prime}(S \tau) \in \mathcal{R}$, hence using the results on $k$ and $k^{\prime}$ from Section 2.3 we can write $k^{\prime}(S \tau)=\sqrt{1-k^{\prime 2}(\tau)}$.

Theorem 2 shows that

and

$$
M\left(k^{\prime}(\tau)\right)=\frac{1}{\theta_{00}^{2}(\tau)}
$$

hence, using Proposition 2, we have

$$
M\left(k^{\prime}(S \tau)\right)=\frac{1}{\theta_{00}^{2}(S \tau)},
$$

$$
\frac{M\left(k^{\prime}(\tau)\right)}{M\left(k^{\prime}(S \tau)\right)}=-i \tau .
$$

We now introduce the function $f_{\tau}$ defined for $z \in \mathcal{R}$ by

$$
f_{\tau}(z)=i M(z)-\tau M\left(\sqrt{1-z^{2}}\right) .
$$

From the preceding discussion, it is clear that $f_{\tau}$ vanishes at $k^{\prime}(\tau)$, hence the idea to use Newton iterations on $f_{\tau}$ in order to evaluate $k^{\prime}$ at $\tau$. In order to show that this is indeed possible, we now give a few results about $f_{\tau}$. 
Proposition 10. The function $f_{\tau}$ is analytic on $\mathcal{R} \backslash\{1\}$.

Proof. This is a direct consequence of the definition of $f_{\tau}$ and of Proposition 8 ,

Proposition 11. For all $\tau \in \mathcal{F}$,

$$
\frac{d f_{\tau}}{d z}\left(k^{\prime}(\tau)\right)=\frac{-2}{\pi \tau \theta_{01}^{2}(\tau) \theta_{10}^{4}(\tau)} .
$$

Proof. Differentiating the definition of $f_{\tau}$, we get

$$
\frac{d f_{\tau}}{d z}(z)=i \frac{d M}{d z}(z)+\frac{\tau z}{\sqrt{1-z^{2}}} \frac{d M}{d z}\left(\sqrt{1-z^{2}}\right),
$$

hence

$$
\frac{d f_{\tau}}{d z}\left(k^{\prime}(\tau)\right)=i \frac{d M}{d z}\left(k^{\prime}(\tau)\right)+\frac{\tau k^{\prime}(\tau)}{k^{\prime}(S \tau)} \frac{d M}{d z}\left(k^{\prime}(S \tau)\right) .
$$

Using the expression for $\frac{d M}{d z}\left(k^{\prime}(\tau)\right)$ obtained in Proposition 9, we get

$$
\frac{d f_{\tau}}{d z}\left(k^{\prime}(\tau)\right)=\frac{4 \theta_{00}^{\prime}(\tau)}{\pi \theta_{00}(\tau) \theta_{01}^{2}(\tau) \theta_{10}^{4}(\tau)}+\frac{4 \tau k^{\prime}(\tau) \theta_{00}^{\prime}(S \tau)}{i \pi k^{\prime}(S \tau) \theta_{00}(S \tau) \theta_{01}^{2}(S \tau) \theta_{10}^{4}(S \tau)} .
$$

Now, differentiating the identity

$$
\theta_{00}^{2}(S \tau)=-i \tau \theta_{00}^{2}(\tau)
$$

gives us

$$
2 \theta_{00}(S \tau) \theta_{00}^{\prime}(S \tau)=-i \tau^{2} \theta_{00}(\tau)\left(\theta_{00}(\tau)+2 \tau \theta_{00}^{\prime}(\tau)\right) .
$$

Using the equalities

$$
k^{\prime}(\tau)=\frac{\theta_{01}^{2}(\tau)}{\theta_{00}^{2}(\tau)}
$$

and

$$
k^{\prime}(S \tau)=k(\tau)=\frac{\theta_{10}^{2}(\tau)}{\theta_{00}^{2}(\tau)},
$$

and the transformation formulae given in Proposition 2, we obtain the announced result.

We now introduce the function $g: \mathcal{R} \backslash\{1\} \rightarrow \mathbb{C}$ defined by

$$
g(z)=\frac{M(z)^{3}}{z\left(1-z^{2}\right)}
$$

for all $z \in \mathcal{R} \backslash\{1\}$.

It is easy to see that $g$ is analytic on $\mathcal{R} \backslash\{1\}$. Now, using the definitions of $k$ and $k^{\prime}$ and Theorem 2 we have, for all $\tau \in \mathcal{F}$,

$$
g\left(k^{\prime}(\tau)\right)=\frac{1}{\theta_{01}^{2}(\tau) \theta_{10}^{4}(\tau)},
$$

hence from Proposition 11

$$
\frac{d f_{\tau}}{d z}\left(k^{\prime}(\tau)\right)=\frac{-2}{\pi \tau} g\left(k^{\prime}(\tau)\right)
$$


5.2. Newton iterations for the function $f_{\tau}$. In this section, we fix $\tau \in \mathcal{F}$, and write $\xi=k^{\prime}(\tau)$.

Since $f_{\tau}$ is analytic on $\mathcal{R}$ and vanishes at $\xi$, Newton iterations on $f_{\tau}$ are a method of choice for the evaluation of $k^{\prime}$ at $\tau$. Classically, this is done by first setting $z_{0}$ to some approximation of $\xi$, then by considering iterations of the form

$$
z_{n+1}=z_{n}-\frac{f_{\tau}\left(z_{n}\right)}{f_{\tau}^{\prime}\left(z_{n}\right)} .
$$

If $z_{0}$ is sufficiently close to $\xi$, then the sequence $\left(z_{n}\right)$ will converge to $\xi$, and the convergence will be quadratic.

It can be proven that if we replace $f_{\tau}^{\prime}\left(z_{n}\right)$ by $f_{\tau}^{\prime}(\xi)$ in each iteration, then the same result holds. However, this is usually not very useful since $\xi$ is precisely the quantity we want to compute. In our case, we consider iterations of the form

$$
z_{n+1}=z_{n}+\frac{\pi \tau f_{\tau}\left(z_{n}\right)}{2 g\left(z_{n}\right)} .
$$

The idea is that, since $z_{n}$ is an approximation of $\xi$, then $\frac{-2}{\pi \tau} g\left(z_{n}\right)$ is an approximation of $\frac{-2}{\pi \tau} g(\xi)=f_{\tau}^{\prime}(\xi)$. More precisely, if we introduce the values $F(\tau)$ and $G(\tau)$ defined for all $\tau \in \mathcal{F}$ by

$$
F(\tau)=\operatorname{Sup}_{n \geq 2}\left|\frac{f_{\tau}^{(n)}(\xi)}{n ! f_{\tau}^{\prime}(\xi)}\right|^{\frac{1}{n-1}}
$$

and

$$
G(\tau)=\operatorname{Sup}_{n \geq 1}\left|\frac{g^{(n)}(\xi)}{n ! g(\xi)}\right|^{\frac{1}{n}},
$$

we have the following result.

Theorem 3. Let $\tau \in \mathcal{F}$ and $\xi=k^{\prime}(\tau)$. Define the sequence $\left(z_{n}\right)$ by $z_{0} \in \mathcal{R} \backslash\{1\}$ and

$$
z_{n+1}=z_{n}+\frac{\pi \tau f_{\tau}\left(z_{n}\right)}{2 g\left(z_{n}\right)}
$$

for all $n \in \mathbb{N}$. Then, if

$$
\left|\xi-z_{0}\right| \leq \frac{1}{6 \operatorname{Max}(F(\tau), G(\tau))}
$$

and

$$
\left|\xi-z_{0}\right|<\frac{1}{2} \operatorname{Min}(|\xi|,|1-\xi|),
$$

the sequence $\left(z_{n}\right)$ is well defined and we have, for all $n \geq 0$,

$$
\left|\xi-z_{n}\right| \leq\left(\frac{1}{2}\right)^{2^{n}-1}\left|\xi-z_{0}\right| .
$$

In order to prove this theorem, we first prove the following lemma.

Lemma 1. Let $\tau \in \mathcal{F}, \xi=k^{\prime}(\tau)$, and $\alpha=\operatorname{Max}(F(\tau), G(\tau))$. Then for all $z \in \mathcal{R} \backslash\{1\}$ such that

$$
2 \alpha|\xi-z|<1,
$$

\footnotetext{
${ }^{3}$ The fact that $F(\tau)$ and $G(\tau)$ are indeed finite follows from the analyticity of $f_{\tau}$ and $g$.
} 
we have

$$
\left|\xi-z+\frac{\pi \tau f_{\tau}(z)}{2 g(z)}\right| \leq \frac{2 \alpha|z-\xi|^{2}}{1-2 \alpha|z-\xi|}
$$

Proof. Let $\tau \in \mathcal{F}, \xi=k^{\prime}(\tau)$, and $\alpha=\operatorname{Max}(F(\tau), G(\tau))$. We have

$$
\begin{aligned}
\left|\xi-z+\frac{\pi \tau f_{\tau}(z)}{2 g(z)}\right|= & \left|\frac{\pi \tau}{2 g(z)}\right| \cdot\left|f_{\tau}(z)-(z-\xi)\left(\frac{-2}{\pi \tau} g(z)\right)\right| \\
\leq & \left|\frac{\pi \tau}{2 g(z)}\right|\left(\mid f_{\tau}(z)-(z-\xi)\left(\frac{-2}{\pi \tau} g(\xi) \mid\right)\right. \\
& \left.+|z-\xi| \cdot\left|\frac{2}{\pi \tau}\right| \cdot|g(z)-g(\xi)|\right) \\
\leq & \left|\frac{g(\xi)}{g(z)}\right|\left(\frac{\left|f_{\tau}(z)-(z-\xi) f_{\tau}^{\prime}(\xi)\right|}{\left|f_{\tau}^{\prime}(\xi)\right|}+|x-\xi| \cdot \frac{|g(z)-g(\xi)|}{|g(\xi)|}\right) .
\end{aligned}
$$

Now, consider the Taylor expansion for $f_{\tau}$ at $\xi$ :

$$
f_{\tau}(z)=(z-\xi) f_{\tau}^{\prime}(\xi)+\sum_{n \geq 2} \frac{f_{\tau}^{(n)}(\xi)}{n !}(z-\xi)^{n}
$$

hence

$$
\begin{aligned}
\left|\frac{f_{\tau}(z)-(z-\xi) f_{\tau}^{\prime}(\xi)}{f_{\tau}^{\prime}(\xi)}\right| & \leq \sum_{n \geq 2}\left|\frac{f_{\tau}^{(n)}(\xi)}{n ! f_{\tau}^{\prime}(\xi)}\right| \cdot|z-\xi|^{n} \\
& \leq \sum_{n \geq 2} F(\tau)^{n-1}|z-\xi|^{n} \\
& \leq \frac{F(\tau)|z-\xi|^{2}}{1-F(\tau)|z-\xi|}
\end{aligned}
$$

since, by hypothesis, $F(\tau)|z-\xi|<1$.

In a similar fashion, by considering the Taylor expansion for $g$ at $\xi$ and using the definition of $G(\tau)$, we get

$$
\frac{g(z)}{g(\xi)}=1+\sum_{n \geq 1} \frac{g^{(n)}(\xi)}{n ! g(\xi)}(z-\xi)^{n}
$$

where

$$
\left|\sum_{n \geq 1} \frac{g^{(n)}(\xi)}{n ! g(\xi)}(z-\xi)^{n}\right| \leq \frac{G(\tau)|z-\xi|}{1-G(\tau)|z-\xi|} .
$$

The hypothesis on $|z-\xi|$ implies that

$$
\frac{G(\tau)|z-\xi|}{1-G(\tau)|z-\xi|}<1
$$

hence we get

$$
\left|\frac{g(\xi)}{g(z)}\right| \leq \frac{1}{1-\frac{G(\tau)|z-\xi|}{1-G(\tau)|z-\xi|}}=\frac{1-G(\tau)|z-\xi|}{1-2 G(\tau)|z-\xi|}
$$


These inequalities, combined with the fact that the functions $x \mapsto \frac{x}{1-x}$ and $x \mapsto \frac{1-x}{1-2 x}$ are increasing on $[0,1 / 2[$ and the definition of $\alpha$, prove that

$$
\left|\xi-z+\frac{\pi \tau f_{\tau}(z)}{2 g(z)}\right| \leq \frac{2 \alpha|z-\xi|^{2}}{1-2 \alpha|z-\xi|}
$$

Proof of Theorem 3. Let $\tau \in \mathcal{F}, \xi=k^{\prime}(\tau), \alpha=\operatorname{Max}(F(\tau), G(\tau))$, and $z_{0} \in \mathcal{R} \backslash\{1\}$ such that

- $6 \alpha\left|\xi-z_{0}\right|<1$,

- $\left|\xi-z_{0}\right|<\left|z_{0}\right|$,

- $\left|\xi-z_{0}\right|<\left|1-z_{0}\right|$.

We define the sequence $\left(z_{n}\right)$ by

$$
z_{n+1}=z_{n}+\frac{\pi \tau f_{\tau}\left(z_{n}\right)}{2 g\left(z_{n}\right)}
$$

for all $n \in \mathbb{N}$, and let $\lambda=2 \alpha\left|\xi-z_{0}\right|$.

We now prove, by induction on $n$, that for all $n \in \mathbb{N}, z_{n} \in \mathcal{R} \backslash 1$ (i.e., the sequence is well defined), and

$$
\left|\xi-z_{n}\right| \leq\left(\frac{\lambda}{1-\lambda}\right)^{2^{n}-1}\left|\xi-z_{0}\right|
$$

This is clearly true for $n=0$, we now show that if it holds for $n$, then it holds for $n+1$.

Since $z_{n} \in \mathcal{R} \backslash\{1\}$, then $z_{n+1}$ is well defined. Since $6 \alpha\left|\xi-z_{0}\right|<1$, we have $\frac{\lambda}{1-\lambda}<1$, hence $\left|\xi-z_{n}\right| \leq\left|\xi-z_{0}\right|$, and Lemma 1 can be applied to $z_{n}$ to show that

$$
\left|\xi-z_{n+1}\right| \leq \frac{2 \alpha\left|\xi-z_{n}\right|^{2}}{1-2 \alpha\left|\xi-z_{n}\right|}
$$

Combining this with the fact that equation (4) holds for $z_{n}$, we get

$$
\begin{aligned}
\left|\xi-z_{n+1}\right| & \leq \frac{2 \alpha}{1-2 \alpha\left|\xi-z_{n}\right|}\left(\left(\frac{\lambda}{1-\lambda}\right)^{2^{n}-1}\left|\xi-z_{0}\right|\right)^{2} \\
& \leq \frac{2 \alpha}{1-2 \alpha\left|\xi-z_{n}\right|}\left(\frac{\lambda}{1-\lambda}\right)^{2^{n+1}-2}\left|\xi-z_{0}\right|^{2} \\
& \leq\left(\frac{\lambda}{1-\lambda}\right)^{2^{n+1}-1}\left|\xi-z_{0}\right| .
\end{aligned}
$$

Now, the condition $6 \alpha\left|\xi-z_{0}\right|<1$ ensures that $\frac{\lambda}{1-\lambda}<\frac{1}{2}$, hence

$$
\left|\xi-z_{n+1}\right| \leq\left(\frac{1}{2}\right)^{2^{n+1}-1}\left|\xi-z_{0}\right| \leq\left|\xi-z_{0}\right|
$$

Since $\left|\xi-z_{0}\right|<\frac{|\xi|}{2}$ and $\left|\xi-z_{0}\right|<\frac{|1-\xi|}{2}$, then $z_{n+1}$ lies in $\mathcal{R} \backslash\{1\}$, and the theorem is proved. 


\section{Algorithms and COMPlexity Results for the EVAluation of $k^{\prime}$}

6.1. Evaluation of the AGM. In this section, we let $z \in \mathcal{R}$ and we denote by $\left(a_{n}\right)$ and $\left(b_{n}\right)$ the sequences associated with the computation of $M(z)$ (letting $a_{0}=1$ and $\left.b_{0}=z\right)$.

We begin by giving an upper bound on the number of AGM iterations required in order to compute $M(z)$ to a given relative precision.

Proposition 12. For all $z \in \mathcal{R}, N \in \mathbb{N}$, if we denote by $\left(a_{n}\right)$ and $\left(b_{n}\right)$ the sequences associated with the computation of $M(z)$ (with $a_{0}=1$ and $b_{0}=z$ ) and define $n_{N}$ by

$$
n_{N}=\operatorname{Max}\left(\left\lceil\log _{2}\left|\log _{2}\right| z||\right\rceil, 1\right)+\left\lceil\log _{2}(N+3)-1\right\rceil,
$$

then $a_{n_{N}}$ is an approximation of $M(z)$ to relative precision $N$ bits.

Proof. Let $z \in \mathcal{R}$ and $N \geq 0$. As usual, we denote by $\left(a_{n}\right)$ and $\left(b_{n}\right)$ the AGM sequences associated with the computation of $M(z)$, and we introduce the following auxilliary sequences:

- $\left(m_{n}\right)_{n \in \mathbb{N}}\left(\operatorname{resp} .\left(M_{n}\right)_{n \in \mathbb{N}}\right)$, defined by $m_{n}=\operatorname{Min}\left(\left|a_{n}\right|,\left|b_{n}\right|\right)\left(\right.$ resp. $M_{n}=$ $\left.\operatorname{Max}\left(\left|a_{n}\right|,\left|b_{n}\right|\right)\right)$ for all $n \geq 0$,

- $\left(c_{n}\right)_{n \in \mathbb{N}}$ defined by $c_{n}=M_{n} / m_{n}$ for all $n \geq 0$, and

- $\left(\theta_{n}\right)_{n \in \mathbb{N}}$, where $\theta_{n}$ is the unoriented angle between $a_{n}$ and $b_{n}$.

The sequence $\left(M_{n}\right)$ is clearly decreasing while, from the proof of Proposition 2.1 of [4,

$$
\begin{gathered}
\left|a_{n+1}-b_{n+1}\right| \leq \frac{\left|a_{n}-b_{n}\right|}{2}, \\
m_{n+1} \geq m_{n} \cos \frac{\theta_{n}}{2}, \\
m_{n} \geq \frac{\sin \theta_{0}}{\theta_{0}} m_{0} \geq \frac{2}{\pi} m_{0},
\end{gathered}
$$

and $\theta_{n+1} \leq \frac{\theta_{n}}{2}$ for all $n \geq 0$.

We then have, for $n \geq 0$,

$$
\left|\frac{a_{n+1}}{b_{n+1}}\right|^{2}=\frac{\left|a_{n}+b_{n}\right|^{2}}{4\left|a_{n} b_{n}\right|} \leq \frac{\left(2 M_{n}\right)^{2}}{4 M_{n} m_{n}}=c_{n}
$$

and

$$
\left|\frac{b_{n+1}}{a_{n+1}}\right|^{2}=\frac{4\left|a_{n} b_{n}\right|}{\left|a_{n}+b_{n}\right|^{2}} \leq \frac{4 M_{n} m_{n}}{4 m_{n}^{2} \cos ^{2} \theta_{n}}=\frac{c_{n}}{\cos ^{2} \theta_{n}},
$$

where we used the fact that

$$
\left|a_{n}+b_{n}\right|^{2}=\left|a_{n}\right|^{2}+\left|b_{n}\right|^{2}+2\left|a_{n} b_{n}\right| \cos \theta_{n} \geq 2 m_{n}^{2}\left(1+\cos \theta_{n}\right)=4 m_{n}^{2} \cos ^{2} \theta_{n} .
$$

Using the fact that $\theta_{n} \leq \frac{\pi}{2}$, this proves that

$$
c_{n+1} \leq \sqrt{2 c_{n}} .
$$

For all $n \geq 0$, we have

$$
\left|a_{n+1}-b_{n+1}\right|=\frac{\left|\sqrt{a_{n}}-\sqrt{b_{n}}\right|^{2}}{2}=\frac{\left|a_{n}-b_{n}\right|^{2}}{2\left|\sqrt{a_{n}}+\sqrt{b_{n}}\right|^{2}},
$$


and

$$
\begin{aligned}
\left|\sqrt{a_{n}}+\sqrt{b_{n}}\right|^{2} & =\left|a_{n}\right|+\left|b_{n}\right|+2\left|\sqrt{a_{n}} \sqrt{b_{n}} \cos \frac{\theta_{n}}{2}\right| \\
& \geq 2 m_{n}\left(1+\cos \frac{\theta_{n}}{2}\right)=4 m_{n} \cos ^{2} \frac{\theta_{n}}{4},
\end{aligned}
$$

so (using the fact that $\frac{\theta_{n}}{4} \leq \frac{\pi}{8}$ ),

$$
\left|a_{n+1}-b_{n+1}\right| \leq \frac{\left|a_{n}-b_{n}\right|^{2}}{\alpha m_{n}}
$$

where $\alpha=8 \cos ^{2} \frac{\pi}{8}=4\left(1+\frac{1}{\sqrt{2}}\right)$.

Now, let $n_{0}=\operatorname{Max}\left(\left\lceil\log _{2}\left|\log _{2}\right| z||\right\rceil, 1\right)$ : since $\log _{2} c_{0}=\left|\log _{2}\right| z||$, then (by an induction using equation (8) $) \log _{2} c_{n_{0}} \leq 1$, hence $c_{n_{0}} \leq 2$. Moreover,

$\left|a_{n_{0}}-b_{n_{0}}\right|^{2}=\left|a_{n_{0}}\right|^{2}+\left|b_{n_{0}}\right|^{2}-2\left|a_{n_{0}} b_{n_{0}}\right| \cos \theta_{n_{0}}=M_{n_{0}}^{2}+m_{n_{0}}^{2}-2 M_{n_{0}} m_{n_{0}} \cos \theta_{n_{0}}$,

so that, if we use the facts that $\theta_{n_{0}} \leq \frac{\pi}{4}$ and that the function $x \mapsto x^{2}+1-\sqrt{2} x$ is increasing on $[1,2]$,

$$
\frac{\left|a_{n_{0}}-b_{n_{0}}\right|}{m_{n_{0}}}=\sqrt{c_{n_{0}}^{2}+1-2 c_{n_{0}} \cos \theta_{n_{0}}} \leq \sqrt{c_{n_{0}}^{2}+1-\sqrt{2} c_{n_{0}}} \leq \sqrt{5-2 \sqrt{2}} .
$$

A direct induction using equations (9) and (7) shows that, for all $k \geq 0$,

$$
\log _{2} \frac{\left|a_{n_{0}+k}-b_{n_{0}+k}\right|}{\alpha m_{n_{0}} \frac{\sin \theta_{n_{0}}}{\theta_{n_{0}}}} \leq 2^{k} \log _{2} \frac{\left|a_{n_{0}}-b_{n_{0}}\right|}{\alpha m_{n_{0}} \frac{\sin \theta_{n_{0}}}{\theta_{n_{0}}}} .
$$

Using equation (10) (and the fact that $\frac{\sin \theta_{n_{0}}}{\theta_{n_{0}}} \geq \frac{2 \sqrt{2}}{\pi}$ ), we find that

$$
\log _{2} \frac{\left|a_{n_{0}}-b_{n_{0}}\right|}{\alpha m_{n_{0}} \frac{\sin \theta_{n_{0}}}{\theta_{n_{0}}}} \leq-2,
$$

so that (by equation (11)) for all $k \geq 0$,

$$
\frac{\left|a_{n_{0}+k}-b_{n_{0}+k}\right|}{m_{n_{0}} \frac{\sin \theta_{n_{0}}}{\theta_{n_{0}}}} \leq \frac{\alpha}{2^{2^{k+1}}} \leq \frac{1}{2^{2^{k+1}-3}} .
$$

We are now ready to prove the announced result. Using equation (5), one proves that for all $n \geq 0,\left|M(z)-a_{n}\right| \leq\left|a_{n}-b_{n}\right|$, and equation (7) shows that $|M(z)| \geq$ $m_{n} \frac{\sin \theta_{n}}{\theta_{n}}$ for all $n$, so that

$$
\frac{\left|M(z)-a_{n_{0}+k}\right|}{|M(z)|} \leq \frac{\left|a_{n_{0}+k}-b_{n_{0}+k}\right|}{m_{n_{0}} \frac{\sin \theta_{n_{0}}}{\theta_{n_{0}}}} \leq \frac{1}{2^{2^{k+1}-3}} .
$$

If we set $n_{1}=n_{0}+\left\lceil\log _{2}(N+3)-1\right\rceil$, then the above equation proves that $a_{n_{1}}$ is indeed an approximation of $M(z)$ with relative precision $N$ bits.

This shows that Algorithm 3 can be used for the evaluation of $M(z)$.

Note that, using Newton iterations, square roots can be extracted to relative precision $N$ bits in time $O(\mathcal{M}(N))[3$. 


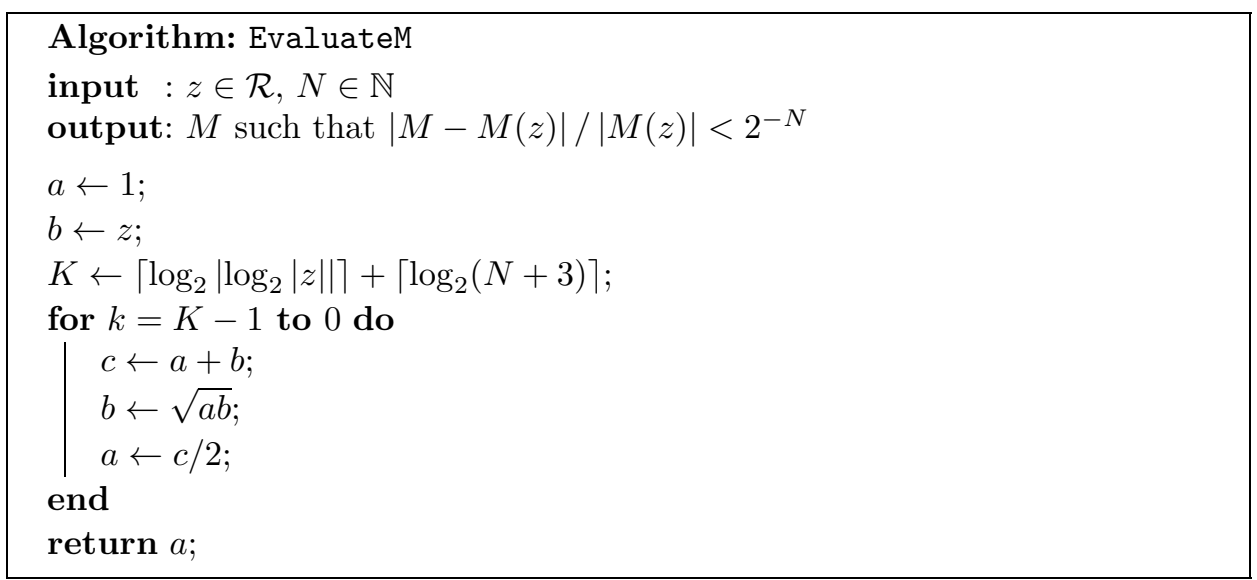

Algorithm 3: Evaluation of the univariate AGM function $M$

We now discuss precision. It is easy to see that only a constant number of bits of relative precision can be lost at each iteration, hence it is sufficient to work with a relative precision of

$$
N+2+2 n_{N}=O(N+\log |\log | z||) \text { bits, }
$$

with $n_{N}$ as defined in Proposition 12,

This leads to the following result.

Corollary 1. The time complexity of algorithm EvaluateM is in

$$
O(\mathcal{M}(N+\log |\log | z||)(\log |\log | z||+\log N)) .
$$

If we suppose that $z$ lies in a compact subset of $\mathcal{R}$, then the complexity is in

$$
O(\mathcal{M}(N) \log N) \text {. }
$$

6.2. Evaluating $k^{\prime}$ at a fixed $\tau \in \mathcal{F}$. In this section, we fix some $\tau \in \mathcal{F}$ and study the complexity of the evaluation of $k^{\prime}$ at $\tau$ as a function of the required relative precision $N$. Our algorithm is based on Theorem [3. Since precision considerations will play an important role in this algorithm and its complexity, we begin by investigating them, before describing the algorithm itself.

6.2.1. Precision considerations. We first note that if $k^{\prime}(\tau)$ is needed to relative precision $N$ bits, the remark from the beginning of Section 5 shows that it is sufficient to compute an approximation to absolute precision $N+4$ bits.

To evaluate $k^{\prime}$, we will use Newton iterations on the function $f_{\tau}$. The validity of our algorithm will be based on the following (slight) variant of Theorem 3 .

Proposition 13. Let $\tau \in \mathcal{F}$ and let $\left(z_{n}\right)$ and $\left(z_{n}^{\prime}\right)$ be two sequences such that

$$
\left|k^{\prime}(\tau)-z_{0}\right| \leq \operatorname{Min}\left(\frac{1}{10 F(\tau)}, \frac{1}{10 G(\tau)}, \frac{\left|k^{\prime}(\tau)\right|}{4}, \frac{\left|1-k^{\prime}(\tau)\right|}{4}\right),
$$

$z_{0}^{\prime}=z_{0}$ and for all $n \geq 1$,

$$
\left|z_{n}-z_{n}^{\prime}\right| \leq\left(\frac{1}{2}\right)^{2^{n}}\left|k^{\prime}(\tau)-z_{0}\right|
$$


and

$$
z_{n+1}^{\prime}=z_{n}+\frac{\pi \tau f_{\tau}\left(z_{n}\right)}{2 g\left(z_{n}\right)} .
$$

Then, for all $n \geq 0$, we have

$$
\left|k^{\prime}(\tau)-z_{n}\right| \leq\left(\frac{1}{2}\right)^{2^{n}-1}\left|k^{\prime}(\tau)-z_{0}\right| .
$$

Proof. The proof mimics that of Theorem 3 .

The interest of this proposition is that the sequence $\left(z_{n}\right)$ used in Theorem 3 does converge to $k^{\prime}(\tau)$, but cannot be computed exactly (since algorithms can only handle numbers with finite precision). Here, the elements of the sequence $\left(z_{n}\right)$ approximate those of the sequence $\left(z_{n}^{\prime}\right)$, hence our algorithm will use the sequence $\left(z_{n}\right)$.

If we define $b_{\tau}$ by

$$
b_{\tau}=\log _{2}\left(\operatorname{Max}\left(10 F(\tau), 10 G(\tau), \frac{4}{\left|k^{\prime}(\tau)\right|}, \frac{4}{\left|1-k^{\prime}(\tau)\right|}\right)\right),
$$

then Proposition 13 tells us that if $z_{0}$ is an approximation of $k^{\prime}(\tau)$ to absolute precision $b_{\tau}$ bits and if, for all $n \geq 0, z_{n+1}$ is an approximation of

$$
z_{n}+\frac{\pi \tau f_{\tau}\left(z_{n}\right)}{2 g\left(z_{n}\right)}
$$

to absolute precision at least $2^{n+1}+b_{\tau}$ bits, then for all $n \geq 0, z_{n}$ is an approximation of $k^{\prime}(\tau)$ to absolute precision $2^{n}-1+b_{\tau}$ bits. Our algorithm will compute such a sequence $\left(z_{n}\right)$ to approximate $k^{\prime}(\tau)$.

We now study the precision at which computations have to be carried out to compute $z_{n+1}$ from $z_{n}$. We have to compute

$$
\frac{\pi \tau f_{\tau}\left(z_{n}\right)}{2 g\left(z_{n}\right)}
$$

to absolute precision $2^{n+1}+1+b_{\tau}$ bits (since one bit of precision can be lost when summing with $z_{n}$ ).

Since $z_{n}$ is an approximation of $k^{\prime}(\tau)$ with absolute precision higher than $b_{\tau}$ bits, then, using the definitions of $b_{\tau}, F(\tau)$, and $G(\tau)$ and Taylor expansions as in the proof of Lemma 1 we get

$$
\frac{-2}{\pi \tau} g\left(z_{n}\right)=\frac{d f_{\tau}}{d z}\left(k^{\prime}(\tau)\right)\left(1+\varepsilon_{g}\right)
$$

and

$$
f_{\tau}\left(z_{n}\right)=\left(z_{n}-k^{\prime}(\tau)\right) \frac{d f_{\tau}}{d z}\left(k^{\prime}(\tau)\right)\left(1+\varepsilon_{f}\right),
$$

with $\left|\varepsilon_{g}\right| \leq 1 / 5$ and $\left|\varepsilon_{f}\right| \leq 1 / 5$, hence

$$
\left|\frac{\pi \tau f_{\tau}\left(z_{n}\right)}{2 g\left(z_{n}\right)}\right| \leq 2\left|\left(k^{\prime}(\tau)-z_{n}\right)\right| \leq \frac{1}{2^{2^{n}+b_{\tau}}} .
$$

Moreover, using the fact that

$$
g\left(k^{\prime}(\tau)\right)=\frac{1}{\theta_{01}^{2}(\tau) \theta_{10}^{4}(\tau)},
$$


it is easy to prove (by considering the definitions of $\theta_{01}$ and $\theta_{10}$ as $q$-series and the fact that $\operatorname{Im}(\tau) \geq \sqrt{3} / 2$ since $\tau \in \mathcal{F})$ that

$$
\frac{1}{10|q|} \geq\left|g\left(k^{\prime}(\tau)\right)\right| \geq \frac{1}{25} .
$$

Taking into account the fact that a few bits of precision can be lost in arithmetic operations, this shows that $f_{\tau}\left(z_{n}\right)$ should be computed to absolute precision

$$
2^{n+1}+4+b_{\tau}+\log _{2}\left(\frac{25 \pi|\tau|}{2}\right) \leq 2^{n+1}+10+b_{\tau}+\log _{2}(|\tau|) \text { bits }
$$

and $g\left(z_{n}\right)$ to absolute precision

$$
2^{n}+4+\log _{2}\left(\frac{1}{10|q|}\right) \leq 2^{n}+1-4 \operatorname{Im}(\tau) \text { bits }
$$

or to relative precision $2^{n}+3$ bits.

6.2.2. The algorithm. From the discussion above, and taking into account the few bits of precision that can be lost through arithmetic operations, Algorithm 4 can be used to evaluate $k^{\prime}(\tau)$.

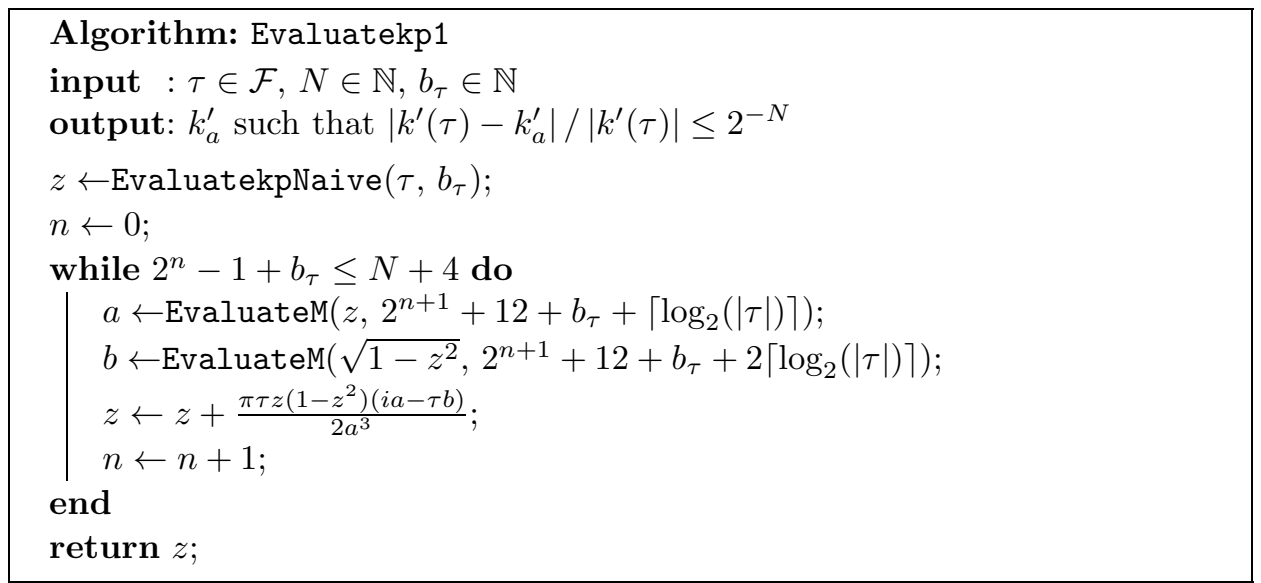

Algorithm 4: Evaluation of $k^{\prime}$ using Newton iterations and the AGM

Since the precision at which the computations have to be carried out increases (it more or less doubles at each Newton iteration), the total running time for this algorithm is proportional to the running time for the last Newton iteration, where $n$ will be on the order of $\log _{2} N$. The cost of that last iteration is, using results from the last section on the complexity of the evaluation of the function $M$, in $O(\mathcal{M}(N) \log N)$. Thus we have proven the following theorem.

Theorem 4. If $\tau$ is fixed in $\mathcal{F}$, then there exists an algorithm that evaluates $k^{\prime}(\tau)$ with relative precision $N$ bits in time $O(\mathcal{M}(N) \log N)=O\left(N^{1+\epsilon}\right)$. 
6.3. Evaluating $k^{\prime}$ at any $\tau \in \mathcal{F}$. In this section we let $\tau$ vary in $\mathcal{F}$ and study the complexity of the evaluation of $k^{\prime}$ at $\tau$ as a function of $\tau$ and of the required relative precision $N$. This is motivated mainly by class polynomial computation, a natural application of our algorithms.

If we consider the algorithm introduced in the last section, then its running time when $\tau$ varies will now also depend on $\tau$ and not only on the required precision $N$. In fact two major problems arise when $\operatorname{Im}(\tau)$ increases:

- The value of $b_{\tau}$ increases as well (the study of the second order derivative of $f_{\tau}$ at $k^{\prime}(\tau)$ shows that $b_{\tau}$ increases at least linearly with $\left.\operatorname{Im}(\tau)\right)$. This is not too important, since the naïve algorithm (Algorithm 2) takes a constant number of multiplications to evaluate $k^{\prime}$ at $\tau$ with a precision linear in $\operatorname{Im}(\tau)$, as remarked in Section 3.2 .

- $k^{\prime}(\tau)$ will tend to 1 , hence $\sqrt{1-k^{\prime}(\tau)^{2}}=k(\tau)$ will tend to 0 . It is in fact equivalent to $4 q^{1 / 2}$, and if we use algorithm Evaluatekp1 (Algorithm 4) to evaluate $k^{\prime}(\tau)$, then the computation of $f_{\tau}$ at each step will require the computation of $M\left(\sqrt{1-z_{n}^{2}}\right)$, and since $\sqrt{1-z_{n}^{2}}$ is on the order of $k(\tau)$, this computation will require a number of AGM iterations in $O(\operatorname{Im}(\tau)+N)$, where $N$ is the required precision.

Due to these reasons, the running time of the algorithm described above, if $\tau$ is not fixed in $\mathcal{F}$, is in $O(\mathcal{M}(N+\log \operatorname{Im}(\tau))(N+\log \operatorname{Im}(\tau)))$. We now consider a variant of that algorithm improving on its complexity.

The basic idea is that, if $k^{\prime}(\tau)$ is known, then $\theta_{00}^{2}(\tau)$ can be computed as $\theta_{00}^{2}(\tau)=1 / M\left(k^{\prime}(\tau)\right)$, and since $k^{\prime}(\tau)=\theta_{01}^{2}(\tau) / \theta_{00}^{2}(\tau)$, so can $\theta_{01}^{2}(\tau)$. Now, using the duplication formulae for the theta constants given in Section 2.2, $\theta_{00}^{2}\left(2^{n} \tau\right)$ and $\theta_{01}^{2}\left(2^{n} \tau\right)$ can also be computed, for all $n \geq 1$, using $n$ AGM iterations. This gives an algorithm with running time in $O(\mathcal{M}(N+n) n)$ for the computation of $k^{\prime}\left(2^{n} \tau\right)$ with precision $N$ bits, from $k^{\prime}(\tau)$.

We now define, for $r>1$,

$$
\mathcal{F}_{r}=\mathcal{F} \cap\{\tau \in \mathbb{C}:|\tau| \leq r\} .
$$

For all $r>1, \mathcal{F}_{r}$ is a compact subset of $\mathcal{F}$, and $F(\tau)$ and $G(\tau)$ are indeed bounded on $\mathcal{F}_{r}$, as a consequence of the following proposition.

Proposition 14. Let $U$ be an open subset of $\mathbb{C}, h$ be an analytic function on $U$ and $K$ be a compact subset of $U$ where $h$ does not vanish. Then the function $H$ defined on $U$ by

$$
H(z)=\operatorname{Sup}_{n \geq 1}\left|\frac{h^{(n)}(z)}{n ! h(z)}\right|^{\frac{1}{n}}
$$

is bounded on $K$.

Proof. Since $K \subset U$, there exists $\varepsilon>0$ such that for all $z \in K$ and $z^{\prime} \in \mathbb{C}$,

$$
\left|z^{\prime}-z\right| \leq \varepsilon \Rightarrow z^{\prime} \in U .
$$

Then, by the Residue Theorem, for all $z \in K$ and $n \geq 1$,

$$
h^{(n)}(z)=\frac{1}{2 \pi i} \int_{C(z, \varepsilon)} \frac{h(t)}{(t-z)^{n+1}} d t,
$$


where $C(z, \varepsilon)$ is the circle of radius $\varepsilon$ centered at $z$. If we let $h_{m}$ (resp. $h_{M}$ ) denote the maximum (resp. the minimum) of the function $|h(z)|$ on $K$, we then have

$$
\left|\frac{h^{(n)}(z)}{n ! h(z)}\right| \leq \frac{h_{M}}{n ! \varepsilon^{n} h_{m}} \leq \frac{h_{M}}{\varepsilon^{n} h_{m}},
$$

for all $n \geq 1$, hence

$$
H(z) \leq \operatorname{Sup}_{n \geq 1} \frac{1}{\varepsilon}\left(\frac{h_{M}}{h_{m}}\right)^{\frac{1}{n}} \leq \frac{h_{M}}{\varepsilon h_{m}} .
$$

Since $F(\tau)$ and $G(\tau)$ are bounded on $\mathcal{F}_{r}$, there exists $B_{r}$ such that for all $\tau \in \mathcal{F}_{r}$,

$$
b_{\tau} \leq B_{r} .
$$

Using Theorem 4, the above result implies that, for all $r>1$, there exists an algorithm that evaluates $k^{\prime}(\tau)$ with precision $N$ bits for all $\tau \in \mathcal{F}_{r}$ and $N \in \mathbb{N}$ in running time $O(\mathcal{M}(N) \log N)$.

Now, if $\tau \in \mathcal{F}$, there exists $n \leq \operatorname{Im}(\tau)$ such that $\tau / 2^{n} \in \mathcal{F}_{2}$, and $k^{\prime}(\tau)$ can be computed either using the naïve algorithm if the required precision $N$ is less than $n$ (this takes time in $O(\mathcal{M}(N)))$ or by first computing $k^{\prime}\left(\tau / 2^{n}\right)$ and then using $n$ AGM iterations as seen above. This leads to Algorithm 5 .

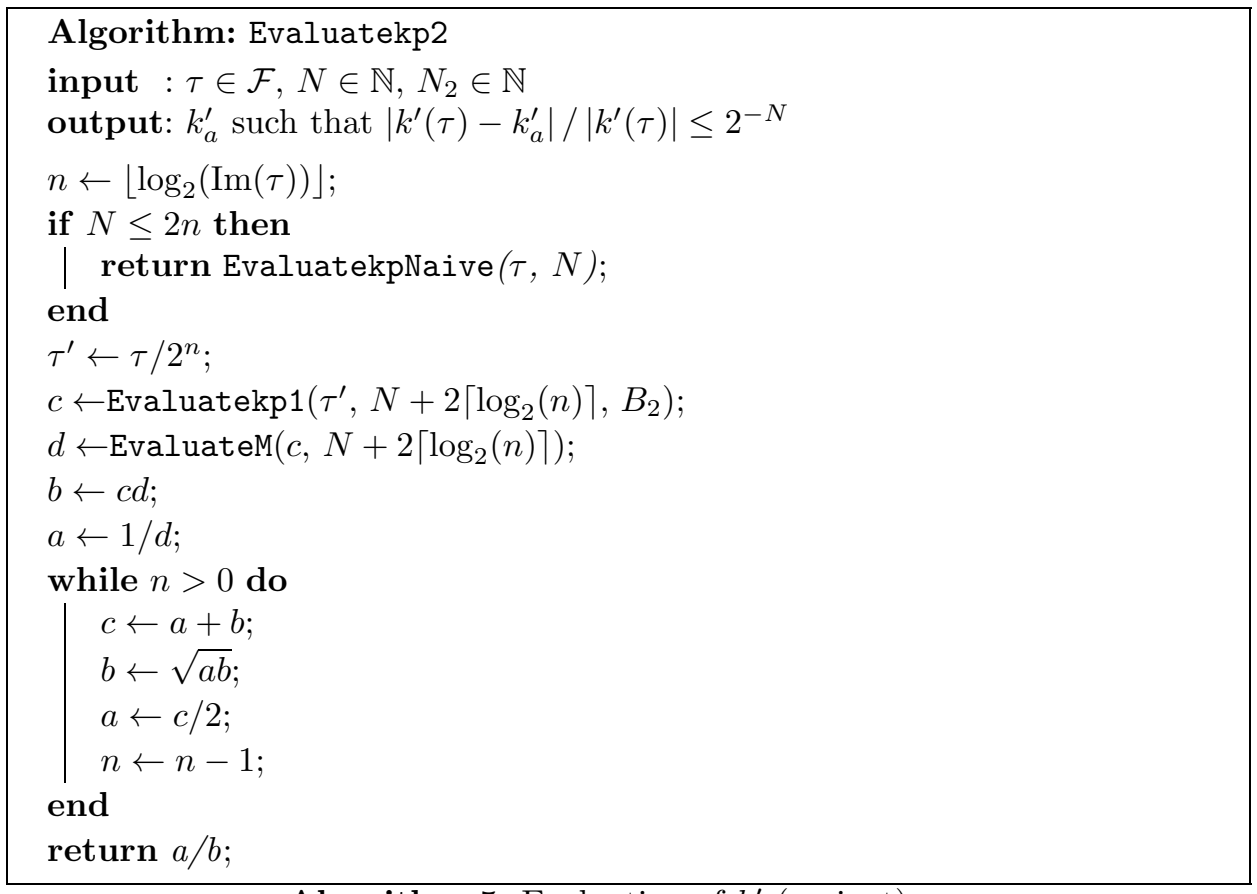

Algorithm 5: Evaluation of $k^{\prime}$ (variant)

Thus we have:

Theorem 5. There exists an algorithm that, for all $N \in \mathbb{N}$, evaluates $k^{\prime}(\tau)$ with relative precision $N$ bits in time $O(\mathcal{M}(N) \log N)=O\left(N^{1+\epsilon}\right)$ for all $\tau \in \mathcal{F}$. 
Note that a direct consequence of this result is that Hilbert class polynomials of degree $h$ can be computed in time $O\left(h^{2+\epsilon}\right)$ using root approximation and reconstruction, as described in [7].

\section{Evaluating other FunCtions}

7.1. Using modular polynomials. It is a well-known fact (see [5] for example) that if $f_{1}$ and $f_{2}$ are two modular functions for two subgroups (of finite index) $\Gamma_{1}$ and $\Gamma_{2}$ of $\Gamma$, then there exists a polynomial $\Phi_{f_{1}, f_{2}} \in \mathbb{C}[X, Y]$ such that, for all $\tau \in \mathcal{H}$

$$
\Phi_{f_{1}, f_{2}}\left(f_{1}(\tau), f_{2}(\tau)\right)=0
$$

Such a polynomial is called a modular polynomial. As for the functions $k^{\prime}$ and $j$ (for which a modular polynomial is in fact given by equation (1)), it is often the case with "classical" modular functions that the modular polynomial linking them together can be chosen with coefficients in $\mathbb{Z}, \mathbb{Q}$ or some finite extension of $\mathbb{Q}$.

We now let $f$ be a modular function for a subgroup $\Gamma^{\prime}$ of $\Gamma$, and we suppose that a modular polynomial $\Phi_{f, k^{\prime}}$ linking $f$ to $k^{\prime}$ is known to an arbitrary precision. Then for any $\tau \in \mathcal{F}, f$ can be evaluated at $\tau$ by first computing the (univariate) polynomial $\Phi_{f, k^{\prime}}\left(X, k^{\prime}(\tau)\right)$, and then by using Newton iterations on that polynomial. This yields an algorithm in $O(\mathcal{M}(N) \log N)$. The algorithm we gave to evaluate $j$ is nothing but a (very) special case of the latter.

7.2. Evaluating the Dedekind $\eta$ function. The Dedekind $\eta$ function can be defined, for all $\tau \in \mathcal{H}$, by

$$
\begin{aligned}
\eta(\tau) & =q^{\frac{1}{12}} \prod_{n \geq 1}\left(1-q^{2 n}\right) \\
& =q^{\frac{1}{12}}\left(1+\sum_{n \geq 1}(-1)^{n}\left(q^{n(3 n-1)}+q^{n(3 n+1)}\right)\right) .
\end{aligned}
$$

(The equivalence between these two definitions, known as Euler's Pentagonal Number Theorem, is proved, for example, in [14]).

Modular invariants defined using simple or double quotients of $\eta$ functions are often used to compute class polynomials [9]. It is thus of interest to have a fast algorithm to evaluate $\eta$.

We first note that, using Theorem 2 and the very definition of $k^{\prime}\left(k^{\prime}(\tau)=\right.$ $\left.\theta_{01}^{2}(\tau) / \theta_{00}^{2}(\tau)\right), \theta_{00}$ and $\theta_{01}$ can be evaluated at any $\tau \in \mathcal{F}$ in time $O(\mathcal{M}(N) \log N)$.

Now, using [16, pages 112-114], we have

$$
\theta_{00}(\tau)=\eta(\tau) f^{2}(\tau)
$$

where $f$ is a modular function satisfying the modular equation

$$
f^{24} k^{\prime 2}\left(1-k^{2}\right)=16 \text {. }
$$

We thus have

$$
\eta^{12}=\frac{k^{\prime 2}\left(1-k^{2}\right) \theta_{00}^{2}}{16}
$$

and it is clear that this equation can be used to evaluate $\eta$ in time $O(\mathcal{M}(N) \log N)$ (the 12-th root can be extracted using Newton iterations). 


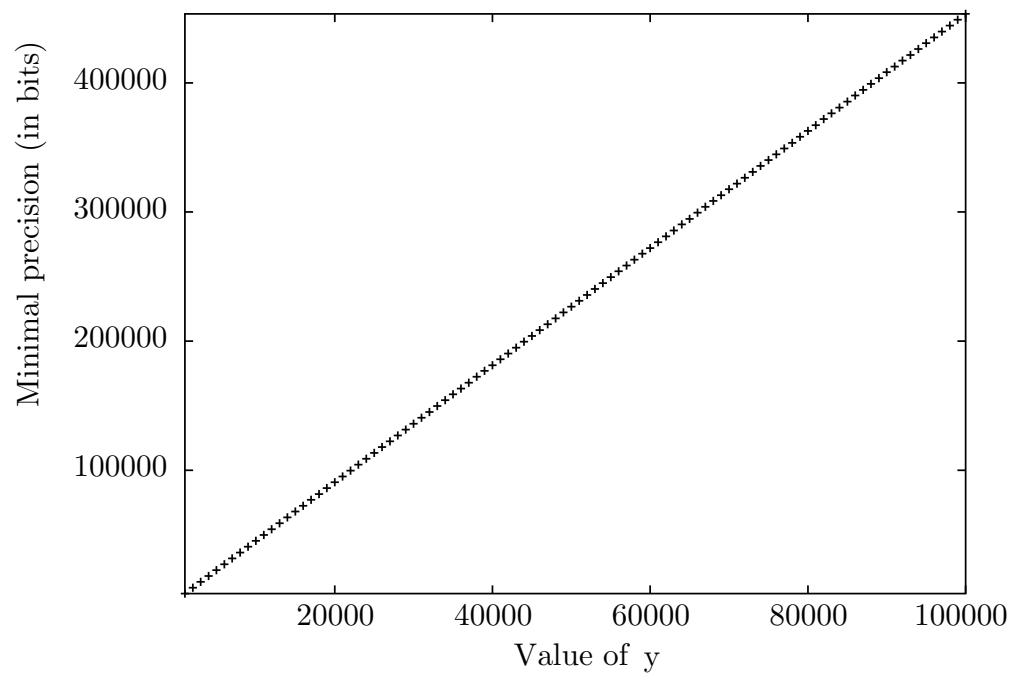

Figure 1. Minimal precision needed to initialize algorithm Evaluatekp1 at $k^{\prime}(0.25+y i)$

\section{EXPERIMENTAL RESULTS}

8.1. Precision needed in order to initialize Newton iterations on $f_{\tau}$. One of the problems is that the value of $b_{\tau}$, which is an input to Algorithm 4 is not known, hence the value of $B_{\tau}$, which is necessary for Algorithm 5 is not known either.

After having carried out a number of numerical experiments, we conjecture that for all $\tau \in \mathcal{F}$,

$$
b_{\tau} \leq 100+\pi \log _{2}(e) \operatorname{Im}(\tau) \leq 100+4.6 \operatorname{Im}(\tau) .
$$

In order to give evidence for this conjecture, we computed the minimal precision at which the initial approximation of $k^{\prime}(\tau)$ has to be computed so that the Newton iterations do indeed converge. Our experiments showed that that quantity mainly depends on $\operatorname{Im}(\tau)$ and not much on $\operatorname{Re}(\tau)$. Figure 1 shows how this minimal precision changes as $\operatorname{Im}(\tau)$ changes (while $\operatorname{Re}(\tau)$ is fixed, equal to 0.25 )This data shows that the minimal precision increases roughly as $4.53 \operatorname{Im}(\tau)$, which corresponds to $\log _{2}(|q|)$ (since $\log _{2}(|q|)=\log _{2}(\exp (-\pi \operatorname{Im}(\tau)))=\pi \log _{2}(e) \operatorname{Im}(\tau)$, and $\left.\pi \log _{2}(e) \simeq 4.5324\right)$. This is in accordance with our conjecture on the value of $b_{\tau}$.

8.2. Timings and comparison with the naïve algorithm. We implemented algorithms Evaluatekp1 and EvaluatekpNaive in C, using the libraries GMP [12], MPFR 13 and MPC [10] for the arithmetic of complex numbers.

For a fixed $\tau$ (here, $\tau=0.123456789+1.23456789 i$ ), the computation time for the evaluation of $k^{\prime}$ using our implementation of algorithm Evaluatekp1 for a required precision up to one million bits (on an Athlon 64 running at $2.4 \mathrm{GHz}$ ) is pictured in Figure 2

Figure 3 shows how our implementation of EvaluatekpNaive compares to that of Evaluatekp1, on the same machine as above. 


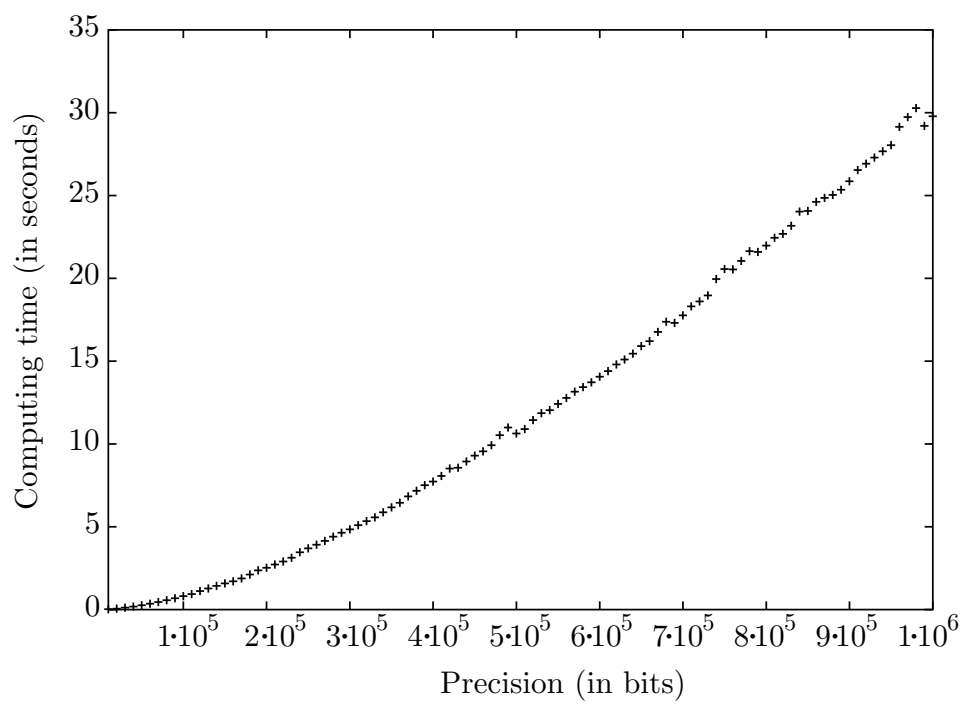

Figure 2. Computing time for algorithm Evaluatekp1

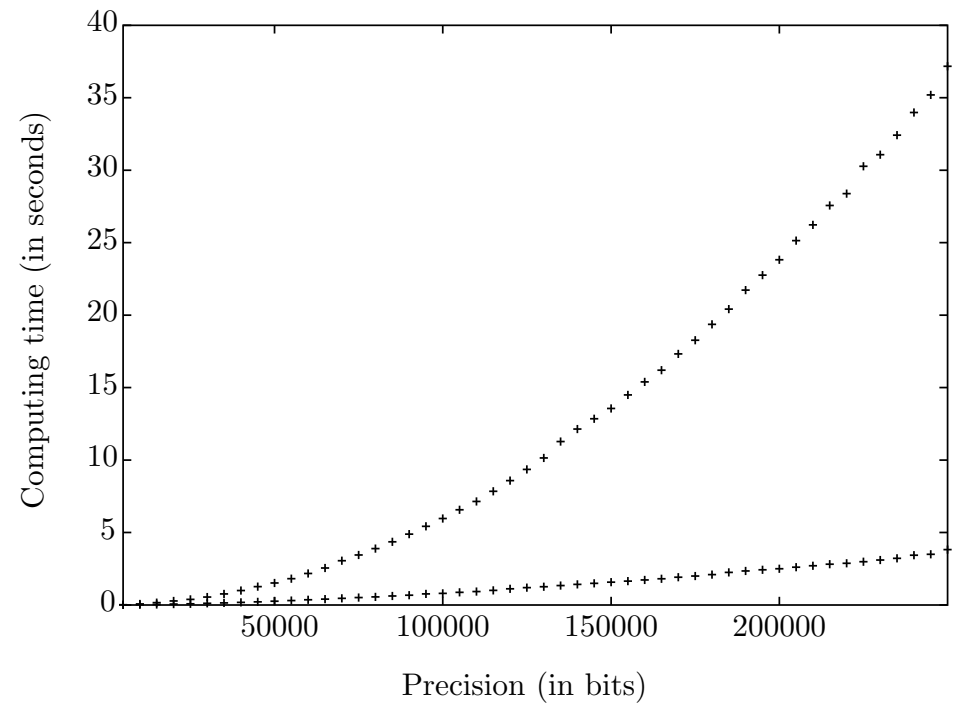

Figure 3. Computing time for algorithms EvaluatekpNaive (top) and Evaluatekp1 (bottom) 


\section{ACKNOWLEDGMENTS}

The author wishes to thank Andreas Enge, Pierrick Gaudry and François Morain for fruitful discussions about this work.

\section{REFERENCES}

[1] H. Baier and G. Köhler. How to compute the coefficients of the elliptic modular function j(z). Experiment. Math., 12(1):115-121, 2001. MR2002678 (2004h:11039)

[2] J. M. Borwein and P. B. Borwein. Pi and the AGM. John Wiley, 1987. MR0877728 (89a:11134)

[3] R. P. Brent. Analytic computational complexity, chapter Multiple-precision zero-finding methods and the complexity of elementary function evaluation, pages 151-176. Academic Press, New York, 1975. MR0423869 (54:11843)

[4] D. A. Cox. The arithmetic-geometric mean of Gauss. Enseign. Math., 30:275-330, 1984. MR0767905 (86a:01027)

[5] M. Deuring. Die Klassenkörper der komplexen Multiplikation. In Enzyklopädie der mathematischen Wissenschaften mit Einschluss ihrer Anwendungen, volume Bd 1, H. 10, T. 2. Teubner, Stuttgart, 1958. MR0167481 (29:4754)

[6] R. Dupont. Borchardt's mean, theta constants in genus 2 and applications, 2005. In preparation.

[7] A. Enge. The complexity of class polynomial computation via floating point approximations, Math. Comp. 78:1089-1107, 2009. MR2476572 (2010h:11097)

[8] A. Enge. The complexity of modular polynomial computation via floating point evaluation and interpolation, 2005. In preparation.

[9] A. Enge and F. Morain. Comparing invariants for class fields of imaginary quadratic fields. In C. Fieker and D. Kohel, editors, Algorithmic Number Theory, volume 2369 of Lecture Notes in Comput. Sci., pages 252-266. Springer-Verlag, 2002. 5th International Symposium, ANTS-V, Sydney, Australia, July 7-12, 2002. Proceedings. MR2041089 (2005a:11179)

[10] A. Enge and P. Zimmermann. MPC - Multiprecision Complex arithmetic library version 0.4, 2004. Available at http://www.loria.fr/ ${ }^{\sim}$ zimmerma/free/.

[11] C. F. Gauss. Werke. Dieterich, Göttingen, 1866-1933.

[12] T. Granlund et al. GMP - GNU Multiprecision library version 4.1, 2002. Available at http://www. swox. com/gmp/.

[13] G. Hanrot, V. Lefèvre, P. Pélissier, and P. Zimmermann. MPFR - Multiple Precision Floating point computations with exact Rounding library version 2.1.0, 2004. Available at http://www.mpfr.org.

[14] D. Mumford. Tata lectures on theta I. Birkhauser, 1984. MR0688651 (85h:14026)

[15] B. Schoeneberg. Elliptic modular functions, volume 203 of Die Grundlehren der mathematischen Wissenschaften in Einzeldarstellungen. Springer-Verlag, 1974. MR0412107 (54:236)

[16] H. Weber. Lehrbuch der Algebra, volume III. Chelsea Publishing Company, New York, 1902.

inRIA Futurs, Projet TANC, Laboratoire LiX, École Polytechnique, 91128 Palaiseau, FRANCE

E-mail address: regis.dupont@m $4 \mathrm{x}$. org 